\title{
On boundary behavior and Dirichlet problem for Beltrami equations
}

\author{
D. Kovtonyuk, I. Petkov, V. Ryazanov and R. Salimov
}

September 26, 2018 (ARXIV-260112-KPRS.tex)

\begin{abstract}
We show that homeomorphic $W_{\text {loc }}^{1,1}$ solutions to the Beltrami equations $\bar{\partial} f=\mu \partial f$ satisfy certain moduli inequalities. On this basis, we develope the theory of the boundary behavior of such solutions and prove a series of criteria for the existence of regular, pseudoregular and multi-valued solutions for the Dirichlet problem to the Beltrami equations in Jordan domains and finitely connected domains.
\end{abstract}

2000 Mathematics Subject Classification: Primary 30C65; Secondary 30C75

Key words: Dirichlet problem, Beltrami equation, moduli inequalities, boundary behavior, homeomorphic, regular, pseudoregular, multi-valued solutions.

\section{Introduction}

Let $D$ be a domain in the complex plane $\mathbb{C}$, i.e., a connected open subset of $\mathbb{C}$, and let $\mu: D \rightarrow \mathbb{C}$ be a measurable function with $|\mu(z)|<1$ a.e. (almost everywhere) in $D$. A Beltrami equation is an equation of the form

$$
f_{\bar{z}}=\mu(z) f_{z}
$$

where $f_{\bar{z}}=\bar{\partial} f=\left(f_{x}+i f_{y}\right) / 2, f_{z}=\partial f=\left(f_{x}-i f_{y}\right) / 2, z=x+i y$, and $f_{x}$ and $f_{y}$ are partial derivatives of $f$ in $x$ and $y$, correspondingly. The function $\mu$ is called the complex coefficient and

$$
K_{\mu}(z)=\frac{1+|\mu(z)|}{1-|\mu(z)|}
$$

the dilatation quotient of the equation (1.1). The Beltrami equation (1.1) is said to be degenerate if ess $\sup K_{\mu}(z)=\infty$. The existence of homeomorphic $W_{\text {loc }}^{1,1}$ solutions was recently established to many degenerate Beltrami equations, see, e.g., related references in the recent monograph [24] and in the surveys [12] and [42].

Boundary value problems for the Beltrami equations are due to the well-known Riemann dissertation in the case of $\mu(z)=0$ and to the papers of Hilbert (1904, 1924) and Poincare (1910) for the corresponding Cauchy-Riemann system. The 
Dirichlet problem was well studied for uniformly elliptic systems, see, e.g., [1] and [45]. The Dirichlet problem for degenerate Beltrami equations in the unit disk was studied in [6]. However, the criteria for the existence of solutions for the Dirichlet problem in [6] are not invariant under conformal mappings of Riemann. Hence we give here the corresponding theorems on the existence of regular solutions in arbitrary Jordan domains as well as of pseudoregular and multi-valued solutions in arbitrary multiply connected domains bounded by a finite collection of mutually disjoint Jordan curves. In comparison with the work [6], our approach is based on estimates of the modulus of dashed lines but not of paths under arbitrary homeomorphic $W_{\text {loc }}^{1,1}$ solutions of the Beltrami equations.

Recall that every holomorphic (analytic) function $f$ in a domain $D \subset \mathbb{C}$ satisfies the simplest Beltrami equation

$$
f_{\bar{z}}=0
$$

with $\mu(z) \equiv 0$. If a holomorphic function $f$ given in the unit disk $\mathbb{D}$ is continuous in its closure, then by the Schwarz formula

$$
f(z)=i \operatorname{Im} f(0)+\frac{1}{2 \pi i} \int_{|\zeta|=1} \operatorname{Re} f(\zeta) \cdot \frac{\zeta+z}{\zeta-z} \frac{d \zeta}{\zeta},
$$

see, e.g., Section 8, Chapter III, Part 3 in [15]. Thus, the holomorphic function $f$ in the unit disk $\mathbb{D}$ is determinated, up to a purely imaginary additive constant $i c, c=\operatorname{Im} f(0)$, by its real part $\varphi(\zeta)=\operatorname{Re} f(\zeta)$ on the boundary of $\mathbb{D}$.

Hence the Dirichlet problem for the Beltrami equation (1.1) in a domain $D \subset \mathbb{C}$ is the problem on the existence of a continuous function $f: D \rightarrow \mathbb{C}$ having partial derivatives of the first order a.e., satisfying (1.1) a.e. and such that

$$
\lim _{z \rightarrow \zeta} \operatorname{Re} f(z)=\varphi(\zeta) \quad \forall \zeta \in \partial D
$$

for a prescribed continuous function $\varphi: \partial D \rightarrow \mathbb{R}$. It is obvious that if $f$ is a solution of this problem, then the function $F(z)=f(z)+i c, c \in \mathbb{R}$, is so.

Here we show that the Dirichlet problem (1.5) has regular solutions in an arbitrary Jordan domain and pseudoregular and multi-valued solutions in an arbitrary finitely connected domain for wide classes of degenerate Beltrami equations (1.1).

Finally, it is necessary to note that the existence of solutions for the Dirichlet problem to the degenerate Beltrami equations with two characteristics

$$
f_{\bar{z}}=\mu(z) f_{z}+\nu(z) \overline{f_{z}}
$$

remains open although the corresponding theorems on the existence of homeomorphic $W_{\text {loc }}^{1,1}$ solutions of (1.6) have been established in the series of the recent papers [2]-[4]. This problem is important because the Beltrami equations of the second type

$$
f_{\bar{z}}=\nu(z) \overline{f_{z}}
$$


play a great role in many problems of mathematical physics, see, e.g., [21]. However, the Dirichlet problem for the equation (1.7) demands an essential modification of our approach.

\section{Preliminaries}

Throughout this paper, $B\left(z_{0}, r\right)=\left\{z \in \mathbb{C}:\left|z_{0}-z\right|<r\right\}, \mathbb{D}=B(0,1), S\left(z_{0}, r\right)=$ $\left\{z \in \mathbb{C}:\left|z_{0}-z\right|=r\right\}, S(r)=S(0, r), R\left(z_{0}, r_{1}, r_{2}\right)=\left\{z \in \mathbb{C}: r_{1}<\left|z-z_{0}\right|<r_{2}\right\}$.

The class BMO was introduced by John and Nirenberg (1961) in the paper [16] and soon became an important concept in harmonic analysis, partial differential equations and related areas; see, e.g., [13] and [40].

Recall that a real-valued function $u$ in a domain $D$ in $\mathbb{C}$ is said to be of bounded mean oscillation in $D$, abbr. $u \in \operatorname{BMO}(D)$, if $u \in L_{\text {loc }}^{1}(D)$ and

$$
\|u\|_{*}:=\sup _{B} \frac{1}{|B|} \int_{B}\left|u(z)-u_{B}\right| d m(z)<\infty,
$$

where the supremum is taken over all discs $B$ in $D, d m(z)$ corresponds to the Lebesgue measure in $\mathbb{C}$ and

$$
u_{B}=\frac{1}{|B|} \int_{B} u(z) d m(z) .
$$

We write $u \in \mathrm{BMO}_{\text {loc }}(D)$ if $u \in \mathrm{BMO}(U)$ for every relatively compact subdomain $U$ of $D$ (we also write $\mathrm{BMO}$ or $\mathrm{BMO}_{\text {loc }}$ if it is clear from the context what $D$ is).

Following the paper [17], see also [18] and [24], we say that a function $\varphi: D \rightarrow$ $\mathbb{R}$ has finite mean oscillation at a point $z_{0} \in D$ if

$$
\varlimsup_{\varepsilon \rightarrow 0} f_{B\left(z_{0}, \varepsilon\right)}\left|\varphi(z)-\widetilde{\varphi}_{\varepsilon}\left(z_{0}\right)\right| d m(z)<\infty
$$

where

$$
\widetilde{\varphi}_{\varepsilon}\left(z_{0}\right)=f_{B\left(z_{0}, \varepsilon\right)} \varphi(z) d m(z)
$$

is the mean value of the function $\varphi(z)$ over the disk $B\left(z_{0}, \varepsilon\right)$. Note that the condition (2.2) includes the assumption that $\varphi$ is integrable in some neighborhood of the point $z_{0}$. We say also that a function $\varphi: D \rightarrow \mathbb{R}$ is of finite mean oscillation in $D$, abbr. $\varphi \in \operatorname{FMO}(D)$ or simply $\varphi \in \operatorname{FMO}$, if $\varphi \in \operatorname{FMO}\left(z_{0}\right)$ for all points $z_{0} \in D$. We write $\varphi \in \operatorname{FMO}(\bar{D})$ if $\varphi$ is given in a domain $G$ in $\mathbb{C}$ such that $\bar{D} \subset G$ and $\varphi \in \operatorname{FMO}\left(z_{0}\right)$ for all $z_{0} \in \bar{D}$.

The following statement is obvious by the triangle inequality.

Proposition 2.1. If, for a collection of numbers $\varphi_{\varepsilon} \in \mathbb{R}, \varepsilon \in\left(0, \varepsilon_{0}\right]$,

$$
\varlimsup_{\varepsilon \rightarrow 0} f_{B\left(z_{0}, \varepsilon\right)}\left|\varphi(z)-\varphi_{\varepsilon}\right| d m(z)<\infty
$$


then $\varphi$ is of finite mean oscillation at $z_{0}$.

In particular choosing in Proposition [2.1, $\varphi_{\varepsilon} \equiv 0, \varepsilon \in\left(0, \varepsilon_{0}\right]$, we obtain the following.

Corollary 2.1. If, for a point $z_{0} \in D$,

$$
\varlimsup_{\varepsilon \rightarrow 0} f_{B\left(z_{0}, \varepsilon\right)}|\varphi(z)| d m(z)<\infty,
$$

then $\varphi$ has finite mean oscillation at $z_{0}$.

Recall that a point $z_{0} \in D$ is called a Lebesgue point of a function $\varphi: D \rightarrow \mathbb{R}$ if $\varphi$ is integrable in a neighborhood of $z_{0}$ and

$$
\lim _{\varepsilon \rightarrow 0} f_{B\left(z_{0}, \varepsilon\right)}\left|\varphi(z)-\varphi\left(z_{0}\right)\right| d m(z)=0 .
$$

It is known that, almost every point in $D$ is a Lebesgue point for every function $\varphi \in L^{1}(D)$. Thus we have by Proposition 2.1 the following corollary.

Corollary 2.2. Every locally integrable function $\varphi: D \rightarrow \mathbb{R}$, has a finite mean oscillation at almost every point in D.

Remark 2.1. Note that the function $\varphi(z)=\log (1 /|z|)$ belongs to BMO in the unit disk $\Delta$, see, e.g., [40], p. 5, and hence also to FMO. However, $\widetilde{\varphi}_{\varepsilon}(0) \rightarrow$ $\infty$ as $\varepsilon \rightarrow 0$, showing that condition (2.5) is only sufficient but not necessary for a function $\varphi$ to be of finite mean oscillation at $z_{0}$. Clearly, $\operatorname{BMO}(D) \subset$ $\mathrm{BMO}_{\text {loc }}(D) \subset \operatorname{FMO}(D)$ and as well-known $\mathrm{BMO}_{\text {loc }} \subset L_{\text {loc }}^{p}$ for all $p \in[1, \infty)$, see, e.g., [5], by FMO is not a subclass of $L_{\mathrm{loc}}^{p}$ for any $p>1$ but only $L_{\mathrm{loc}}^{1}$. Thus the class FMO is much more wide than $\mathrm{BMO}_{\text {loc }}$.

Versions of the next lemma has been first proved for the class BMO in the planar case in [32], [33], and then in the space case in [22], [23]. For the FMO case, see the papers [17], [18], [31], [34], [35], and the monograph [24].

Lemma 2.1. Let $D$ be a domain in $\mathbb{C}$ and let $\varphi: D \rightarrow \mathbb{R}$ be a non-negative function of the class $\operatorname{FMO}\left(z_{0}\right)$ for some $z_{0} \in D$. Then

$$
\int_{\varepsilon<\left|z-z_{0}\right|<\varepsilon_{0}} \frac{\varphi(z) d m(z)}{\left(\left|z-z_{0}\right| \log \frac{1}{\left|z-z_{0}\right|}\right)^{2}}=O\left(\log \log \frac{1}{\varepsilon}\right) \quad \text { as } \quad \varepsilon \rightarrow 0
$$

for some $\varepsilon_{0} \in\left(0, \delta_{0}\right)$ where $\delta_{0}=\min \left(e^{-e}, d_{0}\right), d_{0}=\sup _{z \in D}\left|z-z_{0}\right|$.

Recall a connection between some integral conditions, see, e.g., [37]-[39].

Theorem 2.1. Let $Q: \mathbb{D} \rightarrow[0, \infty]$ be a measurable function such that

$$
\int_{\mathbb{D}} \Phi(Q(z)) d m(z)<\infty
$$


where $\Phi:[0, \infty] \rightarrow[0, \infty]$ is a non-decreasing convex function such that

$$
\int_{\delta_{0}}^{\infty} \frac{d \tau}{\tau \Phi^{-1}(\tau)}=\infty
$$

for some $\delta_{0}>\Phi(0)$. Then

$$
\int_{0}^{1} \frac{d r}{r q(r)}=\infty
$$

where $q(r)$ is the average of the function $Q(z)$ over the circle $|z|=r$.

The following lemma is also useful, see Lemma 2.1 in [20] or Lemma 9.2 in [24].

Lemma 2.2. Let $(X, \mu)$ be a measure space with a finite measure $\mu, p \in(1, \infty)$ and let $\varphi: X \rightarrow(0, \infty)$ be a measurable function. Set

$$
I(\varphi, p)=\inf _{\alpha} \int_{X} \varphi \alpha^{p} d \mu
$$

where the infimum is taken over all measurable functions $\alpha: X \rightarrow[0, \infty]$ such that

$$
\int_{X} \alpha d \mu=1 .
$$

Then

$$
I(\varphi, p)=\left[\int_{X} \varphi^{-\lambda} d \mu\right]^{-\frac{1}{\lambda}}
$$

where

$$
\lambda=\frac{q}{p}, \quad \frac{1}{p}+\frac{1}{q}=1,
$$

i.e. $\lambda=1 /(p-1) \in(0, \infty)$. Moreover, the infimum in (2.11) is attained only for the function

$$
\alpha_{0}=C \cdot \varphi^{-\lambda}
$$

where

$$
C=\left(\int_{X} \varphi^{-\lambda} d \mu\right)^{-1} .
$$

Finally, recall that the (conformal) modulus of a family $\Gamma$ of paths $\gamma$ in $\mathbb{C}$ is the quantity

$$
M(\Gamma)=\inf _{\varrho \in \operatorname{adm} \Gamma} \int_{\mathbb{C}} \varrho^{2}(z) d m(z)
$$


where a Borel function $\varrho: \mathbb{C} \rightarrow[0, \infty]$ is admissible for $\Gamma$, write $\varrho \in \operatorname{adm} \Gamma$, if

$$
\int_{\gamma} \varrho d s \geqslant 1 \quad \forall \gamma \in \Gamma .
$$

Here $s$ is a natural parameter of the length on $\gamma$.

\section{On regular domains}

First of all, recall the following topological notion. A domain $D \subset \mathbb{C}$ is said to be locally connected at a point $z_{0} \in \partial D$ if, for every neighborhood $U$ of the point $z_{0}$, there is a neighborhood $V \subseteq U$ of $z_{0}$ such that $V \cap D$ is connected. If this condition holds for all $z_{0} \in \partial D$, then $D$ is said to be locally connected on $\partial D$. Note that every Jordan domain $D$ in $\mathbb{C}$ is locally connected on $\partial D$, see, e.g., [46], p. 66.

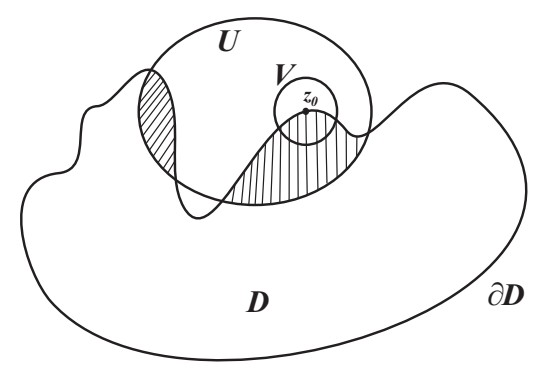

We say that $\partial D$ is weakly flat at a point $z_{0} \in \partial D$ if, for every neighborhood $U$ of the point $z_{0}$ and every number $P>0$, there is a neighborhood $V \subset U$ of $z_{0}$ such that

$$
M(\Delta(E, F ; D)) \geqslant P
$$

for all continua $E$ and $F$ in $D$ intersecting $\partial U$ and $\partial V$. We say that $\partial D$ is weakly flat if it is weakly flat at each point $z_{0} \in \partial D$.

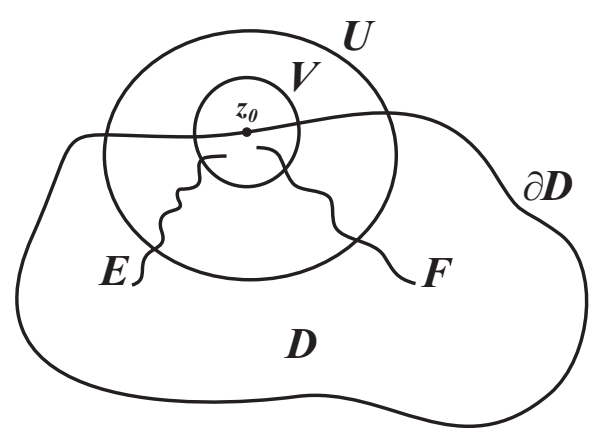

We also say that a point $z_{0} \in \partial D$ is strongly accessible if, for every neighborhood $U$ of the point $z_{0}$, there exist a compactum $E$ in $D$, a neighborhood $V \subset U$ of $z_{0}$ and a number $\delta>0$ such that

$$
M(\Delta(E, F ; D)) \geqslant \delta
$$


for all continua $F$ in $D$ intersecting $\partial U$ and $\partial V$. We say that $\partial D$ is strongly accessible if each point $z_{0} \in \partial D$ is strongly accessible.

Here, in the definitions of strongly accessible and weakly flat boundaries, one can take as neighborhoods $U$ and $V$ of a point $z_{0}$ only balls (closed or open) centered at $z_{0}$ or only neighborhoods of $z_{0}$ in another fundamental system of neighborhoods of $z_{0}$. These conceptions can also be extended in a natural way to the case of $\overline{\mathbb{C}}$ and $z_{0}=\infty$. Then we must use the corresponding neighborhoods of $\infty$.

It is easy to see that if a domain $D$ in $\mathbb{C}$ is weakly flat at a point $z_{0} \in \partial D$, then the point $z_{0}$ is strongly accessible from $D$. Moreover, it was proved by us that if a domain $D$ in $\mathbb{C}$ is weakly flat at a point $z_{0} \in \partial D$, then $D$ is locally connected at $z_{0}$, see, e.g., Lemma 5.1 in [20] or Lemma 3.15 in [24].

The notions of strong accessibility and weak flatness at boundary points of a domain in $\mathbb{C}$ defined in [19], see also [20] and [31], are localizations and generalizations of the corresponding notions introduced in [22] and [23], cf. with the properties $P_{1}$ and $P_{2}$ by Väisälä in [44] and also with the quasiconformal accessibility and the quasiconformal flatness by Näkki in [29]. Many theorems on a homeomorphic extension to the boundary of quasiconformal mappings and their generalizations are valid under the condition of weak flatness of boundaries. The condition of strong accessibility plays a similar role for a continuous extension of the mappings to the boundary.

A domain $D \subset \mathbb{C}$ is called a quasiextremal distance domain, abbr. QEDdomain, see [10], if

$$
M(\Delta(E, F ; \mathbb{C}) \leqslant K \cdot M(\Delta(E, F ; D))
$$

for some $K \geqslant 1$ and all pairs of nonintersecting continua $E$ and $F$ in $D$.

It is well known, see, e.g., Theorem 10.12 in [44], that

$$
M(\Delta(E, F ; \mathbb{C})) \geqslant \frac{2}{\pi} \log \frac{R}{r}
$$

for any sets $E$ and $F$ in $\mathbb{C}$ intersecting all the circles $S\left(z_{0}, \rho\right), \rho \in(r, R)$. Hence a QED-domain has a weakly flat boundary. One example in [24], Section 3.8, shows that the inverse conclusion is not true even in the case of simply connected domains in $\mathbb{C}$.

A domain $D \subset \mathbb{C}$ is called a uniform domain if each pair of points $z_{1}$ and $z_{2} \in D$ can be joined with a rectifiable curve $\gamma$ in $D$ such that

$$
s(\gamma) \leqslant a \cdot\left|z_{1}-z_{2}\right|
$$

and

$$
\min _{i=1,2} s\left(\gamma\left(z_{i}, z\right)\right) \leqslant b \cdot \operatorname{dist}(z, \partial D)
$$


for all $z \in \gamma$ where $\gamma\left(z_{i}, z\right)$ is the portion of $\gamma$ bounded by $z_{i}$ and $z$, see [25]. It is known that every uniform domain is a QED-domain but there exist QEDdomains that are not uniform, see [10]. Bounded convex domains and bounded domains with smooth boundaries are simple examples of uniform domains and, consequently, QED-domains as well as domains with weakly flat boundaries.

In the mapping theory and in the theory of differential equations, it is also often applied the so-called Lipschitz boundaries.

They say that a domain $D$ in $\mathbb{C}$ is Lipschitz if every point $z_{0} \in \partial D$ has a neighborhood $U$ that can be mapped by a bi-Lipschitz homeomorphism $\varphi$ onto the unit disk $\mathbb{D} \subset \mathbb{C}$ in such a way that $\varphi(\partial D \cap U)$ is the intersection of $\mathbb{D}$ with the real axis. Note that a bi-Lipschitz homeomorphism is quasiconformal and the modulus is a quasiinvariant under such mappings. Hence the Lipschitz domains have weakly flat boundaries.

Recall that a map $\varphi: U \rightarrow \mathbb{C}$ is said to be Lipcshitz provided $\left|\varphi\left(z_{1}\right)-\varphi\left(z_{2}\right)\right| \leqslant$ $M \cdot\left|z_{1}-z_{2}\right|$ for some $M<\infty$ and for all $z_{1}$ and $z_{2} \in U$, and bi-Lipcshitz if in addition $M^{*}\left|z_{1}-z_{2}\right| \leqslant\left|\varphi\left(z_{1}\right)-\varphi\left(z_{2}\right)\right|$ for some $M^{*}>0$ and for all $z_{1}$ and $z_{2} \in U$.

\section{On estimates of modulus of dashed lines}

A continuous mapping $\gamma$ of an open subset $\Delta$ of the real axis $\mathbb{R}$ or a circle into $D$ is called a dashed line, see, e.g., 6.3 in [24]. Note that such a set $\Delta$ consists of a countable collection of mutually disjoint intervals in $\mathbb{R}$. This is the motivation for the term. The notion of the modulus of the family $\Gamma$ of dashed lines $\gamma$ is defined similarly to (2.17). We say that a property $P$ holds for a.e. (almost every) $\gamma \in \Gamma$ if a subfamily of all lines in $\Gamma$ for which $P$ fails has the modulus zero, cf. [8]. Later on, we also say that a Lebesgue measurable function $\varrho: \mathbb{C} \rightarrow[0, \infty]$ is extensively admissible for $\Gamma$, write $\varrho \in \operatorname{ext} \operatorname{adm} \Gamma$, if (2.18) holds for a.e. $\gamma \in \Gamma$, see, e.g., Section 9.2 in [24].

Theorem 4.1. Let $f$ be a homeomorphic $W_{\mathrm{loc}}^{1,1}$ solution of the Beltrami equation (1.1) in a domain $D \subseteq \mathbb{C}$. Then

$$
M\left(f \Sigma_{\varepsilon}\right) \geqslant \inf _{\varrho \in \operatorname{ext} \operatorname{adm} \Sigma_{\varepsilon}} \int_{D} \frac{\varrho^{2}(z)}{K_{\mu}(z)} d m(z)
$$

for all $z_{0} \in \bar{D}$, where $\varepsilon \in\left(0, \varepsilon_{0}\right), \varepsilon_{0} \in\left(0, d_{0}\right), d_{0}=\sup _{z \in D}\left|z-z_{0}\right|$ and $\Sigma_{\varepsilon}$ denotes the family of dashed lines consisting of all intersections of the circles $S\left(z_{0}, r\right)$, $r \in\left(\varepsilon, \varepsilon_{0}\right)$, with $D$.

Proof. Let $B$ be a (Borel) set of all points $z$ in $D$ where $f$ has a total differential with $J_{f}(z) \neq 0$. It is known that $B$ is the union of a countable collection of Borel sets $B_{l}, l=1,2, \ldots$, such that $f_{l}=\left.f\right|_{B_{l}}$ is a bi-Lipschitz homeomorphism, see, e.g., Lemma 3.2.2 in [7]. With no loss of generality, we may assume that the $B_{l}$ 
are mutually disjoint. Denote also by $B_{*}$ the set of all points $z \in D$ where $f$ has a total differential with $f^{\prime}(z)=0$.

Note that the set $B_{0}=D \backslash\left(B \cup B_{*}\right)$ has the Lebesgue measure zero in $\mathbb{C}$ by the well known Gehring-Lehto-Menchoff theorem, see [9] and [28]. Hence by Theorem 2.11 in [20], see also Lemma 9.1 in [24], length $\left(\gamma \cap B_{0}\right)=0$ for a.e. paths $\gamma$ in $D$. Let us show that length $\left(f(\gamma) \cap f\left(B_{0}\right)\right)=0$ for a.e. circle $\gamma$ centered at $z_{0} \in \bar{D}$.

The latter follows from absolute continuity of $f$ on closed subarcs of $\gamma \cap D$ for a.e. such circle $\gamma$. Indeed, the class $W_{\text {loc }}^{1,1}$ is invariant with respect to local quasiisometries and the functions in $W_{\text {loc }}^{1,1}$ is absolutely continuous on lines, see, e.g., Theorems 1.1.7 and 1.1.3 in [27], respectively. Applying say the transformation of coordinates $\log \left(z-z_{0}\right)$, we come to the absolute continuity on a.e. such circle $\gamma$. Fix $\gamma_{0}$ on which $f$ is absolutely continuous and length $\left(\gamma_{0} \cap B_{0}\right)=0$. Then length $\left(f(\gamma) \cap f\left(B_{0}\right)\right)=$ length $f\left(\gamma_{0} \cap B_{0}\right)$ and for every $\varepsilon>0$ there is an open set $\omega_{\varepsilon}$ in $\gamma_{0} \cap D$ such that $\gamma_{0} \cap B_{0} \subset \omega_{\varepsilon}$ with length $\omega_{\varepsilon}<\varepsilon$, see, e.g., Theorem $\operatorname{III}(6.6)$ in [41]. The open set $\omega_{\varepsilon}$ consists of a countable collection of open arcs $\gamma_{i}$ of the circle $\gamma_{0}$. By the construction $\sum_{i}$ length $\gamma_{i}<\varepsilon$ and by the absolute continuity of $f$ on $\gamma_{0}$ the sum $\delta:=\sum_{i}$ length $f\left(\gamma_{i}\right)$ is arbitrarily small for small enough $\varepsilon>0$. Hence length $f\left(\gamma_{0} \cap B_{0}\right)=0$.

Thus, length $\left(\gamma_{*} \cap f\left(B_{0}\right)\right)=0$ where $\gamma_{*}=f(\gamma)$ for a.e. circle $\gamma$ centered at $z_{0}$. Now, let $\varrho_{*} \in \operatorname{adm} f(\Gamma)$ where $\Gamma$ is the collection of all dashed lines $\gamma \cap D$ for such circles $\gamma$ and $\varrho_{*} \equiv 0$ outside $f(D)$. Set $\varrho \equiv 0$ outside $D$ and on $B_{0}$ and

$$
\varrho(z):=\varrho_{*}(f(z))\left(\left|f_{z}\right|+\left|f_{\bar{z}}\right|\right) \quad \text { for } z \in B \cup B_{*} .
$$

Arguing piecewise on $B_{l}$, we have by Theorem 3.2.5 under $m=1$ in [7] that

$$
\int_{\gamma} \varrho d s \geqslant \int_{\gamma_{*}} \varrho_{*} d s_{*} \geqslant 1 \quad \text { for a.e. } \gamma \in \Gamma
$$

because length $\left(f(\gamma) \cap f\left(B_{0}\right)\right)=0$ and length $\left(f(\gamma) \cap f\left(B_{*}\right)\right)=0$ for a.e. $\gamma \in \Gamma$, consequently, $\varrho \in \operatorname{ext}$ adm $\Gamma$.

On the other hand, again arguing piecewise on $B_{l}$, we have the inequality

$$
\int_{D} \frac{\varrho^{2}(z)}{K_{\mu}(z)} d m(z) \leqslant \int_{f(D)} \varrho_{*}^{2}(w) d m(w)
$$

because $\varrho(z)=0$ on $B_{*}$. Thus, we obtain (4.1). 
Theorem 4.2. Let $f$ be a homeomorphic $W_{\text {loc }}^{1,1}$ solution of the Beltrami equation (1.1) in a domain $D \subseteq \mathbb{C}$. Then

$$
M\left(f \Sigma_{\varepsilon}\right) \geqslant \int_{\varepsilon}^{\varepsilon_{0}} \frac{d r}{\left\|K_{\mu}\right\|_{1}\left(z_{0}, r\right)}, \quad \forall z_{0} \in \bar{D}, \varepsilon \in\left(0, \varepsilon_{0}\right), \varepsilon_{0} \in\left(0, d_{0}\right),
$$

where $d_{0}=\sup _{z \in D}\left|z-z_{0}\right|, \Sigma_{\varepsilon}$ denotes the family of dashed lines consisting of all the intersections of the circles $S\left(z_{0}, r\right), r \in\left(\varepsilon, \varepsilon_{0}\right)$, with $D$ and

$$
\left\|K_{\mu}\right\|_{1}\left(z_{0}, r\right):=\int_{D\left(z_{0}, r\right)} K_{\mu}(z)|d z|
$$

is the norm in $L_{1}$ of $K_{\mu}$ over $D\left(z_{0}, r\right)=\left\{z \in D:\left|z-z_{0}\right|=r\right\}=D \cap S\left(z_{0}, r\right)$.

Proof. Indeed, for every $\varrho \in \operatorname{ext} \operatorname{adm} \Sigma_{\varepsilon}$,

$$
A_{\varrho}(r)=\int_{D\left(z_{0}, r\right)} \varrho(z)|d z| \neq 0 \quad \text { a.e. in } r \in\left(\varepsilon, \varepsilon_{0}\right)
$$

is a measurable function in the parameter $r$, say by the Fubini theorem. Thus, we may request the equality $A_{\varrho}(r) \equiv 1$ a.e. in $r \in\left(\varepsilon, \varepsilon_{0}\right)$ and

$$
\inf _{\varrho \in \operatorname{ext} \operatorname{adm} \Sigma_{\varepsilon}} \int_{D \cap R_{\varepsilon}} \frac{\varrho^{2}(z)}{K_{\mu}(z)} d m(z)=\int_{\varepsilon}^{\varepsilon_{0}}\left(\inf _{\alpha \in I(r)} \int_{D\left(z_{0}, r\right)} \frac{\alpha^{2}(z)}{K_{\mu}(z)}|d z|\right) d r
$$

where $R_{\varepsilon}=R\left(z_{0}, \varepsilon, \varepsilon_{0}\right)$ and $I(r)$ denotes the set of all measurable functions $\alpha$ on the dashed line $D\left(z_{0}, r\right)=S\left(z_{0}, r\right) \cap D$ such that

$$
\int_{D\left(z_{0}, r\right)} \alpha(z)|d z|=1
$$

Hence Theorem 4.2 follows by Lemma 2.2 with $X=D\left(z_{0}, r\right)$, the length as a measure $\mu$ on $D\left(x_{0}, r\right), \varphi=\left.\frac{1}{K_{\mu}}\right|_{D\left(z_{0}, r\right)}$ and $p=2$.

Later on, the following lemma will be useful, too. Here we use the standard conventions $a / \infty=0$ for $a \neq \infty$ and $a / 0=\infty$ if $a>0$ and $a \cdot \infty=0$, see, e.g., [41], p. 6.

Lemma 4.1. Let $Q: \mathbb{C} \rightarrow(0, \infty)$ be a locally integrable function. Set

$$
\begin{gathered}
\|Q\|_{1}\left(z_{0}, r\right)=\int_{S\left(z_{0}, r\right)} Q(z)|d z|, \quad z_{0} \in \mathbb{C}, r \in(0, \infty), \\
I=\int_{r_{1}}^{r_{2}} \frac{d r}{\|Q\|_{1}\left(z_{0}, r\right)}, \eta_{0}(r)=\frac{1}{I \cdot\|Q\|_{1}\left(z_{0}, r\right)}, \quad r \in\left(r_{1}, r_{2}\right), \quad 0<r_{1}<r_{2}<\infty .
\end{gathered}
$$


Then

$$
I^{-1}=\int_{r_{1}<\left|z-z_{0}\right|<r_{2}} Q(z) \cdot \eta_{0}^{2}\left(\left|z-z_{0}\right|\right) d m(z) \leqslant \int_{r_{1}<\left|z-z_{0}\right|<r_{2}} Q(z) \cdot \eta^{2}\left(\left|z-z_{0}\right|\right) d m(z)
$$

for every measurable function $\eta:\left(r_{1}, r_{2}\right) \rightarrow[0, \infty]$ such that

$$
\int_{r_{1}}^{r_{2}} \eta(r) d r=1
$$

Proof. If $I=\infty$, then the inequality (4.6) is obvious. Note also that $I \neq 0$ because in the contrary case $\|Q\|_{1}\left(z_{0}, r\right)=\infty$ for a.e. $r \in\left(r_{1}, r_{2}\right)$ contradicting the condition $Q \in L_{\text {loc }}^{1}$. Hence we may assume further that $0<I<\infty$. By (4.7), $\eta(r) \neq \infty$ a.e. in $\left(r_{1}, r_{2}\right)$ and we have that $\eta(r)=\alpha(r) w(r)$ a.e. in $\left(r_{1}, r_{2}\right)$ where

$$
\alpha(r)=\|Q\|_{1}\left(z_{0}, r\right) \eta(r), \quad w(r)=\frac{1}{\|Q\|_{1}\left(z_{0}, r\right)} .
$$

By the Fubini theorem in the polar coordinates

$$
C:=\int_{r_{1}<\left|z-z_{0}\right|<r_{2}} Q(z) \cdot \eta^{2}\left(\left|z-z_{0}\right|\right) d m(z)=\int_{r_{1}}^{r_{2}} \alpha^{2}(r) \cdot w(r) d r
$$

By Jensen's inequality with weights $w(r)$, see, e.g., Theorem 2.6.2 in [30] applied to the convex function $\varphi(t)=t^{2}$ in the interval $\Omega=\left(r_{1}, r_{2}\right)$ and to the probability measure

$$
\nu(E)=\frac{1}{I} \int_{E} w(r) d r, \quad E \subset \Omega
$$

we obtain that

$$
\left(f \alpha^{2}(r) w(r) d r\right)^{1 / 2} \geqslant f \alpha(r) w(r) d r=\frac{1}{I}
$$

where we have also used the fact that $\eta(r)=\alpha(r) w(r)$ satisfies (4.7). Thus,

$$
C \geqslant\left(\int_{r_{1}}^{r_{2}} \frac{d r}{\|Q\|_{1}\left(z_{0}, r\right)}\right)^{-1}
$$

and the proof is complete. 


\section{On the boundary behavior of homeomorphic solutions}

Theorem 5.1. Let $D$ and $D^{\prime}$ be domains in $\mathbb{C}$ and let $f: D \rightarrow D^{\prime}$ be a homeomorphic $W_{\text {loc }}^{1,1}$ solution of the Beltrami equation (1.1). Suppose that $D$ is bounded and locally connected on $\partial D$ and $\partial D^{\prime}$ is strongly accessible. If for all $z_{0} \in \partial D$

$$
\int_{0}^{\delta\left(z_{0}\right)} \frac{d r}{\left\|K_{\mu}\right\|_{1}\left(z_{0}, r\right)}=\infty
$$

for some $\delta\left(z_{0}\right) \in\left(0, d\left(z_{0}\right)\right)$ where $d\left(z_{0}\right)=\sup _{z \in D}\left|z-z_{0}\right|$ and

$$
\left\|K_{\mu}\right\|_{1}\left(z_{0}, r\right)=\int_{D \cap S\left(z_{0}, r\right)} K_{\mu}(z)|d z| .
$$

Then $f$ can be extended to $\bar{D}$ by continuity in $\overline{\mathbb{C}}$.

The proof of Theorem 5.1 is reduced to the following lemma.

Lemma 5.1. Let $D$ and $D^{\prime}$ be domains in $\mathbb{C}$ and let $f: D \rightarrow D^{\prime}$ be a homeomorphic $W_{\mathrm{loc}}^{1,1}$ solution of the Beltrami equation (1.1). Suppose that the domain $D$ is bounded and locally connected at $z_{0} \in \partial D$ and $\partial D^{\prime}$ is strongly accessible at least at one point of the cluster set

$$
L:=C\left(z_{0}, f\right)=\left\{y \in \overline{\mathbb{C}}: w=\lim _{k \rightarrow \infty} f\left(z_{k}\right), z_{k} \rightarrow z_{0}\right\}
$$

If the condition (5.1) holds at $z_{0}$, then $f$ extends to $z_{0}$ by continuity in $\overline{\mathbb{C}}$.

Proof. Note that $L \neq \varnothing$ in view of compactness of the extended space $\overline{\mathbb{C}}$. By the condition $\partial D^{\prime}$ is strongly accessible at a point $\zeta_{0} \in L$. Let us assume that there is one more point $z_{0} \in L$ and set $U=B\left(z_{0}, r_{0}\right)$ where $0<r_{0}<\left|\zeta_{0}-z_{0}\right|$.

In view of local connectedness of $D$ at $z_{0}$, there is a sequence of neighborhoods $V_{k}$ of $z_{0}$ with domains $D_{k}=D \cap V_{k}$ and $\delta\left(V_{k}\right) \rightarrow 0$ as $k \rightarrow \infty$. Choose in the domains $D_{k}^{\prime}=f D_{k}$ points $\zeta_{k}$ and $z_{k}$ with $\left|\zeta_{0}-\zeta_{k}\right|<r_{0}$ and $\left|\zeta_{0}-z_{k}\right|>r_{0}, \zeta_{k} \rightarrow \zeta_{0}$ and $z_{k} \rightarrow z_{0}$ as $k \rightarrow \infty$. Let $C_{k}$ be paths connecting $\zeta_{k}$ and $z_{k}$ in $D_{k}^{\prime}$. Note that by the construction $\partial U \cap C_{k} \neq \varnothing$. By the condition of the strong accessibility of the point $\zeta_{0}$ from $D^{\prime}$, there is a compactum $E \subseteq D^{\prime}$ and a number $\delta>0$ such that

$$
M\left(\Delta\left(E, C_{k} ; D^{\prime}\right)\right) \geq \delta
$$

for large $k$. Without loss of generality we may assume that the last condition holds for all $k=1,2, \ldots$. Note that $C=f^{-1} E$ is a compactum in $D^{\prime}$ and hence $\varepsilon_{0}=\operatorname{dist}\left(z_{0}, C\right)>0$.

Let $\Gamma_{\varepsilon}$ be a family of all paths connecting the circles $C_{\varepsilon}=\left\{z \in \mathbb{C}:\left|z-z_{0}\right|=\varepsilon\right\}$ and $C_{0}=\left\{z \in \mathbb{C}:\left|z-z_{0}\right|=\varepsilon_{0}\right\}$ in $D$. Note that $C_{k} \subset f B_{\varepsilon}$ for every fixed 
$\varepsilon \in\left(0, \varepsilon_{0}\right)$ for large $k$ where $B_{\varepsilon}=B\left(z_{0}, \varepsilon\right)$. Thus, $M\left(f \Gamma_{\varepsilon}\right) \geqslant \delta$ for all $\varepsilon \in\left(0, \varepsilon_{0}\right)$. However, by [14] and [47],

$$
M\left(f \Gamma_{\varepsilon}\right) \leqslant \frac{1}{M\left(f \Sigma_{\varepsilon}\right)}
$$

where $\Sigma_{\varepsilon}$ is the family of all dashed lines $D(r):=\left\{z \in D:\left|z-z_{0}\right|=r\right\}$, $r \in\left(\varepsilon, \varepsilon_{0}\right)$. Thus, $M\left(f \Gamma_{\varepsilon}\right) \rightarrow 0$ as $\varepsilon \rightarrow 0$ by Theorem 4.2 in view of (5.1). The contradiction disproves the above assumption.

Combining Lemmas 4.1 and [5.1, we obtain also the following.

Lemma 5.2. Let $D$ and $D^{\prime}$ be domains in $\mathbb{C}, D$ be locally connected on $\partial D$, $\partial D^{\prime}$ be strongly accessible and let $Q: \mathbb{C} \rightarrow(0, \infty)$ be a locally integrable function. Suppose that $f: D \rightarrow D^{\prime}$ is a homeomorphic $W_{\text {loc }}^{1,1}$ solution of the Beltrami equation (1.1) such that $K_{\mu}(z) \leq Q(z)$ a.e. in $D$ and

$$
\int_{\varepsilon<\left|z-z_{0}\right|<\varepsilon_{0}} Q(z) \cdot \psi_{z_{0}, \varepsilon}^{2}\left(\left|z-z_{0}\right|\right) d m(z)=o\left(I_{z_{0}}^{2}(\varepsilon)\right) \quad \text { as } \quad \varepsilon \rightarrow 0 \quad \forall z_{0} \in \partial D
$$

for some $\varepsilon_{0} \in\left(0, \delta_{0}\right)$ where $\delta_{0}=\delta\left(z_{0}\right)=\sup _{z \in D}\left|z-z_{0}\right|$ and $\psi_{z_{0}, \varepsilon}(t)$ is a family of non-negative measurable (by Lebesgue) functions on $(0, \infty)$ such that

$$
I_{z_{0}}(\varepsilon):=\int_{\varepsilon}^{\varepsilon_{0}} \psi_{z_{0}, \varepsilon}(t) d t<\infty \quad \forall \varepsilon \in\left(0, \varepsilon_{0}\right) .
$$

Then $f$ can be extended to $\bar{D}$ by continuity in $\overline{\mathbb{C}}$.

Theorem 5.2. Let $D$ and $D^{\prime}$ be domains in $\mathbb{C}, D$ be locally connected on $\partial D, \partial D^{\prime}$ be strongly accessible and let $\mu: D \rightarrow \mathbb{C}$ be a measurable function with $|\mu(z)|<1$ a.e. such that $K_{\mu}(z) \leqslant Q(z)$ a.e. in $D$ for a function $Q: \mathbb{C} \rightarrow[0, \infty]$ in the class $\operatorname{FMO}\left(z_{0}\right)$ for all $z_{0} \in \bar{D}$. Then every homeomorphic $W_{\text {loc }}^{1,1}$ solution $f: D \rightarrow D^{\prime}$ of the Beltrami equation (1.1) can be extended to $\bar{D}$ by continuity in $\overline{\mathbb{C}}$.

We assume that $K_{\mu}$ is extended by zero outside of $D$ in the following consequence of Theorem 5.2.

Corollary 5.1. Let $D$ and $D^{\prime}$ be domains in $\mathbb{C}, D$ be locally connected on $\partial D$ and $\partial D^{\prime}$ be strongly accessible and let $\mu: D \rightarrow \mathbb{C}$ be a measurable function with $|\mu(z)|<1$ a.e. such that

$$
\varlimsup_{\varepsilon \rightarrow 0} f_{B\left(z_{0}, \varepsilon\right)} K_{\mu}(z) d m(z)<\infty \quad \forall z_{0} \in \partial D .
$$

Then every homeomorphic $W_{\text {loc }}^{1,1}$ solution $f: D \rightarrow D^{\prime}$ of the Beltrami equation (1.1) can be extended to $\bar{D}$ by continuity in $\overline{\mathbb{C}}$. 
Lemma 5.3. Let $D$ and $D^{\prime}$ be domains in $\mathbb{C}, z_{1}$ and $z_{2}$ be distinct points in $\partial D, z_{1} \neq \infty$, and let $f: D \rightarrow D^{\prime}$ be a homeomorphic $W_{\mathrm{loc}}^{1,1}$ solution of the Beltrami equation (1.1). Suppose that the function $K_{\mu}$ is integrable on the dashed lines

$$
D(r)=\left\{z \in D:\left|z-z_{1}\right|=r\right\}=D \cap S\left(z_{1}, r\right)
$$

for some set $E$ of numbers $r<\left|z_{1}-z_{2}\right|$ of a positive linear measure. If $D$ is locally connected at $z_{1}$ and $z_{2}$ and $\partial D^{\prime}$ is weakly flat, then

$$
C\left(z_{1}, f\right) \cap C\left(z_{2}, f\right)=\varnothing .
$$

Proof. Without loss of generality, we may assume that the domain $D$ is bounded. Let $d=\left|z_{1}-z_{2}\right|$. Choose $\varepsilon_{0} \in(0, d)$ and $\varepsilon \in\left(0, \varepsilon_{0}\right)$ such that

$$
E_{0}:=\left\{r \in E: r \in\left(\varepsilon, \varepsilon_{0}\right)\right\}
$$

has a positive measure. The choice is possible because of a countable subadditivity of the linear measure and because of the exhaustion of $E$ by the sets

$$
E_{m}:=\{r \in E: r \in(1 / m, d-1 / m)\} .
$$

Note that each of the circles $S\left(z_{1}, r\right), r \in E_{0}$, separates the points $z_{1}$ and $z_{2}$ in $\mathbb{C}$ and $D(r), r \in E_{0}$, in $D$. Thus, by Theorem 4.2 we have that

$$
M\left(f \Sigma_{\varepsilon}\right)>0
$$

where $\Sigma_{\varepsilon}$ denotes the family of all intersections of $D$ with the circles

$$
S(r)=S\left(z_{1}, r\right)=\left\{z \in \mathbb{C}:\left|z-z_{1}\right|=r\right\}, \quad r \in\left(\varepsilon, \varepsilon_{0}\right) .
$$

For $i=1,2$, let $C_{i}$ be the cluster set $C\left(z_{i}, f\right)$ and suppose that $C_{1} \cap C_{2} \neq \varnothing$. Since $D$ is locally connected at $z_{1}$ and $z_{2}$, there exist neighborhoods $U_{i}$ of $z_{i}$ such that $W_{i}=D \cap U_{i}, i=1,2$ are connected and $U_{1} \subset B\left(z_{1}, \varepsilon\right)$ and $U_{2} \subset \mathbb{C} \backslash B\left(z_{1}, \varepsilon_{0}\right)$. Set $\Gamma=\Delta\left(\overline{W_{1}}, \overline{W_{2}} ; D\right)$. By [14] and [47] and (5.9])

$$
M(f \Gamma) \leqslant \frac{1}{M\left(f \Sigma_{\varepsilon}\right)}<\infty .
$$

Let $\zeta_{0} \in C_{1} \cap C_{2}$. Without loss of generality, we may assume that $\zeta_{0} \neq \infty$ because in the contrary case one can use an additional Möbius transformation. Choose $r_{0}>0$ such that $S\left(\zeta_{0}, r_{0}\right) \cap f W_{1} \neq \varnothing$ and $S\left(\zeta_{0}, r_{0}\right) \cap f W_{2} \neq \varnothing$.

By the condition $\partial D^{\prime}$ is weakly flat and hence, given a finite number $M_{0}>$ $M(f \Gamma)$, there is $r_{*} \in\left(0, r_{0}\right)$ such that

$$
M\left(\Delta\left(E, F ; D^{\prime}\right)\right) \geqslant M_{0}
$$

for all continua $E$ and $F$ in $D^{\prime}$ intersecting the circles $S\left(\zeta_{0}, r_{0}\right)$ and $S\left(\zeta_{0}, r_{*}\right)$. However, these circles can be connected by paths $P_{1}$ and $P_{2}$ in the domains $f W_{1}$ and $f W_{2}$, respectively, and for those paths

$$
M_{0} \leqslant M\left(\Delta\left(P_{1}, P_{2} ; D^{\prime}\right)\right) \leqslant M(f \Gamma) .
$$


The contradiction disproves the above assumption that $C_{1} \cap C_{2} \neq \varnothing$. The proof is complete.

As an immediate consequence of Lemma 5.3, we have the following statement.

Theorem 5.3. Let $D$ and $D^{\prime}$ be domains in $\mathbb{C}, D$ locally connected on $\partial D$ and $\partial D^{\prime}$ weakly flat. If $f: D \rightarrow D^{\prime}$ is a homeomorphic $W_{\text {loc }}^{1,1}$ solution of the Beltrami equation (1.1) with $K_{\mu} \in L^{1}(D)$, then $f^{-1}$ has an extension to $\overline{D^{\prime}}$ by continuity in $\overline{\mathbb{C}}$.

Proof. By the Fubini theorem with notations from Lemma 5.3, the set

$$
E=\left\{r \in(0, d):\left.K_{\mu}\right|_{D(r)} \in L^{1}(D(r))\right\}
$$

has a positive linear measure because $K_{\mu} \in L^{1}(D)$.

Remark 5.1. It is clear that it is even sufficient to assume in Theorem 5.3 that $K_{\mu}$ is integrable only in a neighborhood of $\partial D$.

Moreover, by Lemma 5.3 we obtain also the following conclusion.

Theorem 5.4. Let $D$ and $D^{\prime}$ be domains in $\mathbb{C}, D$ bounded and locally connected on $\partial D$ and $\partial D^{\prime}$ weakly flat, and let $f: D \rightarrow D^{\prime}$ be a homeomorphic $W_{\text {loc }}^{1,1}$ solution of the Beltrami equation (1.1) with the coefficient $\mu$ such that the condition (5.1) holds for all $z_{0} \in \partial D$. Then there is an extension of $f^{-1}$ to $\overline{D^{\prime}}$ by continuity in $\overline{\mathbb{C}}$.

Combining Theorems 5.1 and 5.4 , we obtain the following statement.

Theorem 5.5. Let $D$ and $D^{\prime}$ be domains in $\mathbb{C}$ and let $f: D \rightarrow D^{\prime}$ be a homeomorphic $W_{\mathrm{loc}}^{1,1}$ solution of the Beltrami equation (1.1). Suppose that $D$ is bounded and locally connected on $\partial D$ and $\partial D^{\prime}$ is weakly flat. If the condition (5.1) holds for all $z_{0} \in \partial D$, then $f$ has a homeomorphic extension $\bar{f}: \bar{D} \rightarrow \overline{D^{\prime}}$ by continuity in $\overline{\mathbb{C}}$.

In particular, as a consequence of Theorem 5.5 we obtain in the plane the following generalization of the well-known Gehring-Martio theorem on a homeomorphic extension to the boundary of quasiconformal mappings between QED domains in $\mathbb{R}^{n}, n \geq 2$, cf. [10], see also [26].

Corollary 5.2. Let $D$ and $D^{\prime}$ be bounded domains with weakly flat boundaries in $\mathbb{C}$ and let $f: D \rightarrow D^{\prime}$ be a homeomorphic $W_{\text {loc }}^{1,1}$ solution of the Beltrami equation (1.1). If the condition (5.1) holds at every point $z_{0} \in \partial D$, then $f$ has a homeomorphic extension to $\bar{D}$ by continuity in $\overline{\mathbb{C}}$.

By Theorem 5.5 and Lemma 4.1 we have also the following.

Lemma 5.4. Let $D$ and $D^{\prime}$ be domains in $\mathbb{C}, D$ be locally connected on $\partial D$, $\partial D^{\prime}$ be weakly flat and let $Q: \mathbb{C} \rightarrow(0, \infty)$ be a locally integrable function. Suppose 
that $f: D \rightarrow D^{\prime}$ is a homeomorphic $W_{\text {loc }}^{1,1}$ solution of the Beltrami equation (1.1) such that $K_{\mu}(z) \leq Q(z)$ a.e. in $D$ and

$$
\int_{\varepsilon<\left|z-z_{0}\right|<\varepsilon_{0}} Q(z) \cdot \psi_{z_{0}, \varepsilon}^{2}\left(\left|z-z_{0}\right|\right) d m(z)=o\left(I_{z_{0}}^{2}(\varepsilon)\right) \quad \text { as } \quad \varepsilon \rightarrow 0 \quad \forall z_{0} \in \partial D
$$

for some $\varepsilon_{0} \in\left(0, \delta_{0}\right)$ where $\delta_{0}=\delta\left(z_{0}\right)=\sup _{z \in D}\left|z-z_{0}\right|$ and $\psi_{z_{0}, \varepsilon}(t)$ is a family of non-negative measurable (by Lebesgue) functions on $(0, \infty)$ such that

$$
I_{z_{0}}(\varepsilon):=\int_{\varepsilon}^{\varepsilon_{0}} \psi_{z_{0}, \varepsilon}(t) d t<\infty \quad \forall \varepsilon \in\left(0, \varepsilon_{0}\right) .
$$

Then $f$ has a homeomorphic extension $\bar{f}: \bar{D} \rightarrow \overline{D^{\prime}}$ by continuity in $\overline{\mathbb{C}}$.

Theorem 5.6. Let $D$ and $D^{\prime}$ be domains in $\mathbb{C}, D$ be locally connected on $\partial D$, $\partial D^{\prime}$ be weakly flat and let $\mu: D \rightarrow \mathbb{C}$ be a measurable function with $|\mu(z)|<1$ a.e. such that $K_{\mu}(z) \leqslant Q(z)$ a.e. in $D$ for a function $Q: \mathbb{C} \rightarrow[0, \infty]$ in the class $\operatorname{FMO}\left(z_{0}\right)$ for all $z_{0} \in \bar{D}$. Then every homeomorphic $W_{\text {loc }}^{1,1}$ solution $f: D \rightarrow D^{\prime}$ of the Beltrami equation (1.1) has a homeomorphic extension $\bar{f}: \bar{D} \rightarrow \overline{D^{\prime}}$ by continuity in $\overline{\mathbb{C}}$.

We assume that $K_{\mu}$ is extended by zero outside of $D$ in the following consequence of Theorem 5.6.

Corollary 5.3. Let $D$ and $D^{\prime}$ be domains in $\mathbb{C}, D$ be locally connected on $\partial D$ and $\partial D^{\prime}$ be weakly flat and let $\mu: D \rightarrow \mathbb{C}$ be a measurable function with $|\mu(z)|<1$ a.e. such that

$$
\varlimsup_{\varepsilon \rightarrow 0} f_{B\left(z_{0}, \varepsilon\right)} K_{\mu}(z) d m(z)<\infty \quad \forall z_{0} \in \partial D
$$

Then every homeomorphic $W_{\text {loc }}^{1,1}$ solution $f: D \rightarrow D^{\prime}$ of the Beltrami equation (1.1) has a homeomorphic extension $\bar{f}: \bar{D} \rightarrow \overline{D^{\prime}}$ by continuity in $\overline{\mathbb{C}}$.

\section{On regular solutions for the Dirichlet problem in the Jordan domains}

If $\varphi(\zeta) \not \equiv$ const, then the regular solution of such a problem is a continuous, discrete and open mapping $f: D \rightarrow \mathbb{C}$ of the Sobolev class $W_{\text {loc }}^{1,1}$ with its Jacobian $J_{f}(z)=\left|f_{z}\right|^{2}-\left|f_{\bar{z}}\right|^{2} \neq 0$ a.e. satisfying (1.1) a.e. and the condition (1.5). The regular solution of the Dirichlet problem (1.5) with $\varphi(\zeta) \equiv c, \zeta \in \partial D$, for the Beltrami equation (1.1) is the function $f(z) \equiv c, z \in D$.

Recall that a mapping $f: D \rightarrow \mathbb{C}$ is called discrete if the preimage $f^{-1}(y)$ consists of isolated points for every $y \in \mathbb{C}$, and open if $f$ maps every open set $U \subseteq D$ onto an open set in $\mathbb{C}$. 
In this section, we prove that a regular solution of the Dirichlet problem (1.5) exists for every continuous function $\varphi: \partial D \rightarrow \mathbb{R}$ for wide classes of the degenerate Beltrami equations (1.1) in an arbitrary Jordan domain $D$.

Lemma 6.1. Let $D$ be a Jordan domain in $\mathbb{C}$ and $\mu: D \rightarrow \mathbb{C}$ be a measurable function with $|\mu(z)|<1$ a.e. If $K_{\mu}(z) \leq Q(z)$ a.e. in $D$ where $Q: \mathbb{C} \rightarrow(0, \infty)$ is locally integrable and satisfies the condition (5.12) for all $z_{0} \in \bar{D}$, then the Beltrami equation (1.1) has a regular solution $f$ of the Dirichlet problem (1.5) for each continuous function $\varphi: \partial D \rightarrow \mathbb{R}$.

Proof. Let $F$ be a regular homeomorphic solution of the Beltrami equation (1.1) of the class $W_{\text {loc }}^{1,1}$ that exists by Lemma 4.1 in [36]. Note that $\overline{\mathbb{C}} \backslash D^{*}$, where $D^{*}=F(D)$, cannot consist of the single point $\infty$ because in the contrary case $\partial D^{*}$ would be weakly flat. But then by Lemma $5.4 \mathrm{~F}$ should have a homeomorphic extension to $\bar{D}$ that is impossible because $\partial D$ is not a singleton. Moreover, the domain $D^{*}$ is simply connected, see, e.g., either Lemma 5.3 in [17] or Lemma 6.5 in [24]. Thus, by the Riemann theorem, see, e.g., Theorem II.2.1 in [11], $D^{*}$ can be mapped by a conformal mapping $R$ onto the unit disk $\mathbb{D}$. The mapping $g=R \circ F$ is also a regular homeomorphic solution of the Beltrami equation of the class $W_{\text {loc }}^{1,1}$ that maps $D$ onto $\mathbb{D}$. Furthermore, by Lemma $5.4 \mathrm{~g}$ admits a homeomorphic extension $g_{*}: D \rightarrow \overline{\mathbb{D}}$ because $\mathbb{D}$ has a weakly flat boundary and the Jordan domain $D$ is locally connected on its boundary.

Let us find a solution of the Dirichlet problem (1.5) in the form $f=h \circ g$ where $h$ is an analytic function in $\mathbb{D}$ with the boundary condition

$$
\lim _{z \rightarrow \zeta} \operatorname{Re} h(z)=\varphi\left(g_{*}^{-1}(\zeta)\right) \quad \forall \zeta \in \partial \mathbb{D} .
$$

By the Schwarz formula (see, e.g., Section 8, Chapter III, Part 3 in [15]), the analytic function $h$ with $\operatorname{Im} h(0)=0$ can be calculated in $\mathbb{D}$ through its real part on the boundary:

$$
h(z)=\frac{1}{2 \pi i} \int_{|\zeta|=1} \operatorname{Re} \varphi \circ g_{*}^{-1}(\zeta) \cdot \frac{\zeta+z}{\zeta-z} \cdot \frac{d \zeta}{\zeta} .
$$

We see that the function $f=h \circ g$ is the desired regular solution of the Dirichlet problem (1.5) for the Beltrami equation (1.1).

Choosing in Lemma $6.1 \psi(t)=1 /(t \log (1 / t))$, we obtain by Lemma 2.1 the following result.

Theorem 6.1. Let $D$ be a Jordan domain and $\mu: D \rightarrow \mathbb{C}$ be a measurable function with $|\mu(z)|<1$ a.e. such that $K_{\mu}(z) \leqslant Q(z)$ a.e. in $D$ for a function $Q: \mathbb{C} \rightarrow[0, \infty]$ in $\operatorname{FMO}(\bar{D})$. Then the Beltrami equation (1.1) has a regular solution of the Dirichlet problem (1.5) for each continuous function $\varphi: \partial D \rightarrow \mathbb{R}$. 
Corollary 6.1. In particular, the conclusion of Theorem 6.1 holds if every point $z_{0} \in \bar{D}$ is the Lebesgue point of a locally integrable function $Q: \mathbb{C} \rightarrow[0, \infty]$ such that $K_{\mu}(z) \leqslant Q(z)$ a.e. in $D$.

Further we assume that $K_{\mu}$ is extended by zero outside of $D$.

Corollary 6.2. Let $D$ be a Jordan domain and $\mu: D \rightarrow \mathbb{C}$ be a measurable function with $|\mu(z)|<1$ a.e. such that

$$
\varlimsup_{\varepsilon \rightarrow 0} f_{B\left(z_{0}, \varepsilon\right)} K_{\mu}(z) d m(z)<\infty \quad \forall z_{0} \in \bar{D} .
$$

Then the Beltrami equation (1.1) has a regular solution of the Dirichlet problem (1.5) for each continuous function $\varphi: \partial D \rightarrow \mathbb{R}$.

The following statement is proved similarly to Lemma 6.1 on the basis of Theorem 5.5 instead of Lemma 5.4.

Theorem 6.2. Let $D$ be a Jordan domain in $\mathbb{C}$ and $\mu: D \rightarrow \mathbb{C}$ be a measurable function with $|\mu(z)|<1$ a.e. If $K_{\mu} \in L_{\mathrm{loc}}^{1}(D)$ and satisfies the condition

$$
\int_{0}^{\delta\left(z_{0}\right)} \frac{d r}{\left\|K_{\mu}\right\|_{1}\left(z_{0}, r\right)}=\infty \quad \forall z_{0} \in \bar{D}
$$

for some $\delta\left(z_{0}\right) \in\left(0, d\left(z_{0}\right)\right)$ where $d\left(z_{0}\right)=\sup _{z \in D}\left|z-z_{0}\right|$ and

$$
\left\|K_{\mu}\right\|_{1}\left(z_{0}, r\right)=\int_{D \cap S\left(z_{0}, r\right)} K_{\mu}(z)|d z|,
$$

at each point $z_{0} \in \bar{D}$, then the Beltrami equation (1.1) has a regular solution $f$ of the Dirichlet problem (1.5) for each continuous function $\varphi: \partial D \rightarrow \mathbb{R}$.

Corollary 6.3. Let $D$ be a Jordan domain and $\mu: D \rightarrow \mathbb{C}$ be a measurable function such that

$$
k_{z_{0}}(\varepsilon)=O\left(\log \frac{1}{\varepsilon}\right) \quad \forall z_{0} \in \bar{D}
$$

as $\varepsilon \rightarrow 0$, where $k_{z_{0}}(\varepsilon)$ is the average of the function $K_{\mu}(z)$ over $S\left(z_{0}, \varepsilon\right)$. Then the Beltrami equation (1.1) has a regular solution of the Dirichlet problem (1.5) for each continuous function $\varphi: \partial D \rightarrow \mathbb{R}$.

Remark 6.1. In particular, the conclusion of Corollary 7.3 holds if

$$
K_{\mu}(z)=O\left(\log \frac{1}{\left|z-z_{0}\right|}\right) \quad \text { as } \quad z \rightarrow z_{0} \quad \forall z_{0} \in \bar{D} .
$$

Finally, combining Theorems 2.1 and 6.2 we obtain the following. 
Theorem 6.3. Let $D$ be a Jordan domain and $\mu: D \rightarrow \mathbb{D}$ be a measurable function such that

$$
\int_{D} \Phi\left(K_{\mu}(z)\right) d m(z)<\infty
$$

for a convex non-decreasing function $\Phi:[0, \infty] \rightarrow[0, \infty]$. If

$$
\int_{\delta}^{\infty} \frac{d \tau}{\tau \Phi^{-1}(\tau)}=\infty
$$

for some $\delta>\Phi(0)$. Then the Beltrami equation (1.1) has a regular solution of the Dirichlet problem (1.5) for each continuous function $\varphi: \partial D \rightarrow \mathbb{R}$.

Remark 6.2. By the Stoilow theorem, see, e.g., [43], a regular solution $f$ of the Dirichlet problem (1.5) for the Beltrami equation (1.1) with $K_{\mu} \in L_{\mathrm{loc}}^{1}(D)$ can be represented in the form $f=h \circ F$ where $h$ is an analytic function and $F$ is a homeomorphic regular solution of (1.1) in the class $W_{\text {loc }}^{1,1}$. Thus, by Theorem 5.1 in [38] the condition (6.8) is not only sufficient but also necessary to have a regular solution of the Dirichlet problem (1.5) for an arbitrary Beltrami equation (1.1) with the integral constraints (6.7) for any non-constant continuous function $\varphi: \partial D \rightarrow \mathbb{R}$.

Setting $H(t)=\log \Phi(t)$, note that by Theorem 2.1 in [39] the condition 6.8 is equivalent to each of the conditions

$$
\begin{aligned}
& \int_{\Delta}^{\infty} H^{\prime}(t) \frac{d t}{t}=\infty, \\
& \int_{\Delta}^{\infty} \frac{d H(t)}{t}=\infty
\end{aligned}
$$

and (6.10)

$$
\int_{\Delta}^{\infty} H(t) \frac{d t}{t^{2}}=\infty
$$

for some $\Delta>0$, and

$$
\int_{0}^{\delta} H\left(\frac{1}{t}\right) d t=\infty
$$

for some $\delta>0$,

$$
\int_{\Delta_{*}}^{\infty} \frac{d \eta}{H^{-1}(\eta)}=\infty
$$


for some $\Delta_{*}>H(+0)$. Here, the integral in (6.10) is understood as the LebesgueStieltjes integral and the integrals in (6.8) and (6.11)-(6.13) as the ordinary Lebesgue integrals.

Corollary 6.4. In particular, the conclusion of Theorem 6.3 holds if, for some $\alpha>0$,

$$
\int_{D} e^{\alpha K_{\mu}(z)} d m(z)<\infty .
$$

\section{On pseudoregular solutions in multiply connected do- mains}

As it was first noted by Bojarski, see, e.g., section 6 of Chapter 4 in [45], in the case of multiply connected domains the Dirichlet problem for the Beltrami equation, generally speaking, has no solutions in the class of continuous (simply-valued) functions. Hence it is arose the question: whether the existence of solutions for the Dirichlet problem can be obtained in a wider class for the case? It is turned out to be that this is possible in the class of functions having a certain number of poles at prescribed points in $D$. More precisely, for $\varphi(\zeta) \not \equiv$ const, a pseudoregular solution of the problem is a continuous (in $\overline{\mathbb{C}}=\mathbb{C} \cup\{\infty\}$ ) discrete open mapping $f: D \rightarrow \overline{\mathbb{C}}$ in the class $W_{\text {loc }}^{1,1}$ (outside of these poles) with the Jacobian $J_{f}(z)=\left|f_{z}\right|^{2}-\left|f_{\bar{z}}\right|^{2} \neq 0$ a.e. satisfying (1.1) a.e. and the condition (1.5).

Lemma 7.1. Let $D$ be a bounded domain in $\mathbb{C}$ whose boundary consists of $n \geqslant 2$ mutually disjoint Jordan curves and let $\mu: D \rightarrow \mathbb{C}$ be a measurable function such that $|\mu(z)|<1$ a.e. Suppose that $K_{\mu}(z) \leq Q(z)$ a.e. in $D$ where $Q: \mathbb{C} \rightarrow(0, \infty)$ is locally integrable and satisfies the condition (5.12) for all $z_{0} \in \bar{D}$. Then the Beltrami equation (1.1) has a pseudoregular solution of the Dirichlet problem (1.5) for each continuous function $\varphi: \partial D \rightarrow \mathbb{R}, \varphi(\zeta) \not \equiv$ const, with poles at $n$ prescribed points $z_{i} \in D, i=1, \ldots, n$.

Proof. Let $F$ be a regular homeomorphic solution of the Beltrami equation (1.1) of the class $W_{\text {loc }}^{1,1}$ that exists by Lemma 4.1 in [36]. Consider $D^{*}=f(D)$. Note that $\partial D^{*}$ has $n$ connected components $\Gamma_{i}, i=1, \ldots, n$ that correspond in the natural way to connected components of $\partial D$, the Jordan curves $\gamma_{i}$, see, e.g., either Lemma 5.3 in [17] or Lemma 6.5 in [24].

Thus, by Theorem V.6.2 in [11] the domain $D_{*}$ can be mapped by a conformal map $R$ onto a circular domain $\mathbb{D}_{*}$ whose boundary consists of $n$ circles or points, i.e. $\mathbb{D}_{*}$ has a weakly flat boundary. Note that the mapping $g:=R \circ F$ is a regular homeomorphic solution of the Beltrami equation in the class $W_{\text {loc }}^{1,1}$ admitting a homeomorphic extension $g_{*}: \bar{D} \rightarrow \overline{\mathbb{D}_{*}}$ by Lemma 5.4 . 
Let us find a solution of the Dirichlet problem (1.5) in the form $f=h \circ g$ where $h$ is a meromorphic function with $n$ poles at the prescribed points $w_{i}=g\left(z_{i}\right)$, $i=1, \ldots, n$ in $\mathbb{D}_{*}$ with the boundary condition

$$
\lim _{w \rightarrow \zeta} \operatorname{Re} h(w)=\varphi\left(g_{*}^{-1}(\zeta)\right) \quad \forall \zeta \in \partial \mathbb{D}_{*}
$$

Such a function $h$ exists by theorem 4.14 in [45].

We see that the function $f=h \circ g$ is the desired pseudoregular solution of the Dirichlet problem (1.5) for the Beltrami equation (1.1) with $n$ poles just at these prescribed points $z_{i}, i=1, \ldots, n$.

Arguing, similarly to the last section, by the special choice of the functional parameter $\psi$ in Lemma 7.1, we obtain the following result.

Theorem 7.1. Let $D$ be a bounded domain in $\mathbb{C}$ whose boundary consists of $n \geqslant 2$ mutually disjoint Jordan curves and $\mu: D \rightarrow \mathbb{C}$ be a measurable function such that $|\mu(z)|<1$ a.e., and $K_{\mu}(z) \leqslant Q(z)$ a.e. in $\bar{D}$ for a function $Q: \mathbb{C} \rightarrow$ $[0, \infty]$ in the class $\mathrm{FMO}(\bar{D})$. Then the Beltrami equation (1.1) has a pseudoregular solution of the Dirichlet problem (1.5) for every continuous function $\varphi: \partial D \rightarrow \mathbb{R}$, $\varphi(\zeta) \not \equiv$ const, with poles at $n$ prescribed points in $D$.

Corollary 7.1. In particular, the conclusion of Theorem 7.1 holds if every point $z_{0} \in \bar{D}$ is the Lebesgue point of a locally integrable function $Q: \mathbb{C} \rightarrow[0, \infty]$ such that $K_{\mu}(z) \leqslant Q(z)$ a.e. in $D$.

Corollary 7.2. In particular, the conclusion of Theorem 7.1 holds if

$$
\varlimsup_{\varepsilon \rightarrow 0} f_{B\left(z_{0}, \varepsilon\right)} K_{\mu}(z) d m(z)<\infty \quad \forall z_{0} \in \bar{D} .
$$

As above, here we assume that $K_{\mu}$ is extended by zero outside of $D$.

Theorem 7.2. Let $D$ be a bounded domain in $\mathbb{C}$ whose boundary consists of $n \geqslant 2$ mutually disjoint Jordan curves and $\mu: D \rightarrow \mathbb{C}$ be a measurable function with $|\mu(z)|<1$ a.e. If $K_{\mu} \in L_{\mathrm{loc}}^{1}(D)$ and satisfies the condition

$$
\int_{0}^{\delta\left(z_{0}\right)} \frac{d r}{\left\|K_{\mu}\right\|_{1}\left(z_{0}, r\right)}=\infty \quad \forall z_{0} \in \bar{D}
$$

for some $\delta\left(z_{0}\right) \in\left(0, d\left(z_{0}\right)\right)$ where $d\left(z_{0}\right)=\sup _{z \in D}\left|z-z_{0}\right|$ and

$$
\left\|K_{\mu}\right\|_{1}\left(z_{0}, r\right)=\int_{D \cap S\left(z_{0}, r\right)} K_{\mu}|d z| .
$$

Then the Beltrami equation (1.1) has a pseudoregular solution of the Dirichlet problem (1.5) for every continuous function $\varphi: \partial D \rightarrow \mathbb{R}, \varphi(\zeta) \not \equiv$ const, with poles at $n$ prescribed points in $D$. 
Corollary 7.3. Let $D$ be a bounded domain in $\mathbb{C}$ whose boundary consists of $n \geqslant 2$ mutually disjoint Jordan curves and $\mu: D \rightarrow \mathbb{C}$ be a measurable function such that

$$
k_{z_{0}}(\varepsilon)=O\left(\log \frac{1}{\varepsilon}\right) \quad \text { as } \quad \varepsilon \rightarrow 0 \quad \forall z_{0} \in \bar{D},
$$

where $k_{z_{0}}(\varepsilon)$ is the average of the function $K_{\mu}(z)$ over the circle $\left\{z \in \mathbb{C}:\left|z-z_{0}\right|=\right.$ $\varepsilon\}$. Then the Beltrami equation (1.1) has a pseudoregular solution of the Dirichlet problem (1.5) for every continuous function $\varphi: \partial D \rightarrow \mathbb{R}, \varphi(\zeta) \not \equiv$ const, with poles at $n$ prescribed points in $D$.

Remark 7.1. In particular, the conclusion of Corollary 7.3 holds if

$$
K_{\mu}(z)=O\left(\log \frac{1}{\left|z-z_{0}\right|}\right) \quad \text { as } \quad z \rightarrow z_{0} \quad \forall z_{0} \in \bar{D} .
$$

Theorem 7.3. Let $D$ be a bounded domain in $\mathbb{C}$ whose boundary consists of $n \geqslant 2$ mutually disjoint Jordan curves and let $\mu: D \rightarrow \mathbb{C}$ be a measurable function with $|\mu(z)|<1$ a.e. such that

$$
\int_{D} \Phi\left(K_{\mu}(z)\right) d m(z)<\infty
$$

for a convex non-decreasing function $\Phi:[0, \infty] \rightarrow[0, \infty]$. If

$$
\int_{\delta}^{\infty} \frac{d \tau}{\tau \Phi^{-1}(\tau)}=\infty
$$

for some $\delta>\Phi(0)$. Then the Beltrami equation (1.1) has a pseudoregular solution of the Dirichlet problem (1.5) for each continuous function $\varphi: \partial D \rightarrow \mathbb{R}, \varphi(\zeta) \not \equiv$ const, with poles at $n$ prescribed inner points in $D$.

Corollary 7.4. In particular, the conclusion of Theorem 7.3 holds if, for some $\alpha>0$,

$$
\int_{D} e^{\alpha K_{\mu}(z)} d m(z)<\infty .
$$

\section{On multi-valued solutions in multiply connected do- mains}

In finitely connected domains $D$ in $\mathbb{C}$, in addition to pseudoregular of solutions, the Dirichlet problem (1.5) for the Beltrami equation (1.1) admits multi-valued solutions in the spirit of the theory of multi-valued analytic functions. We say 
that a discrete open mapping $f: B\left(z_{0}, \varepsilon_{0}\right) \rightarrow \mathbb{C}$, where $B\left(z_{0}, \varepsilon_{0}\right) \subseteq D$, is a local regular solution of the equation (1.1) if $f \in W_{\mathrm{loc}}^{1,1}, J_{f}(z) \neq 0$ and $f$ satisfies (1.1) a.e. in $B\left(z_{0}, \varepsilon_{0}\right)$.

The local regular solutions $f: B\left(z_{0}, \varepsilon_{0}\right) \rightarrow \mathbb{C}$ and $f_{*}: B\left(z_{*}, \varepsilon_{*}\right) \rightarrow \mathbb{C}$ of the equation (1.1) will be called extension of each to other if there is a finite chain of such solutions $f_{i}: B\left(z_{i}, \varepsilon_{i}\right) \rightarrow \mathbb{C}, i=1, \ldots, m$, that $f_{1}=f_{0}, f_{m}=f_{*}$ and $f_{i}(z) \equiv f_{i+1}(z)$ for $z \in E_{i}:=B\left(z_{i}, \varepsilon_{i}\right) \cap B\left(z_{i+1}, \varepsilon_{i+1}\right) \neq \emptyset, i=1, \ldots, m-1$. A collection of local regular solutions $f_{j}: B\left(z_{j}, \varepsilon_{j}\right) \rightarrow \mathbb{C}, j \in J$, will be called a multi-valued solution of the equation (1.1) in $D$ if the disks $B\left(z_{j}, \varepsilon_{j}\right)$ cover the whole domain $D$ and $f_{j}$ are extensions of each to other through the collection. A multi-valued solution of the equation (1.1) will be called a multi-valued solution of the Dirichlet problem (1.5) if $u(z)=\operatorname{Re} f(z)=\operatorname{Re} f_{j}(z), z \in B\left(z_{j}, \varepsilon_{j}\right)$, $j \in J$, is a simply-valued function in $D$ satisfying the condition $\lim _{z \in \zeta} u(z)=\varphi(\zeta)$ for all $\zeta \in D$.

Lemma 8.1. Let $D$ be a bounded domain in $\mathbb{C}$ whose boundary consists of $n \geqslant 2$ mutually disjoint Jordan curves and let $\mu: D \rightarrow \mathbb{C}$ be a measurable function such that $|\mu(z)|<1$ a.e. Suppose that $K_{\mu}(z) \leq Q(z)$ a.e. in $D$ where $Q: \mathbb{C} \rightarrow(0, \infty)$ is locally integrable and satisfies (5.12) for all $z_{0} \in \bar{D}$. Then the Beltrami equation (1.1) has a multi-valued solutions of the Dirichlet problem (1.5) for each continuous function $\varphi: \partial D \rightarrow \mathbb{R}$.

Proof. Let $F$ be a regular homeomorphic solution of the Beltrami equation (1.1) in the class $W_{\text {loc }}^{1,1}$ that exists by Lemma 4.1 in [36]. As it was showed under the proof of Lemma [5.4, we may assume that $D_{*}:=F(D)$ is a circular domain and that $F$ can be extended to a homeomorphism $F_{*}: \bar{D} \rightarrow \overline{D_{*}}$. Let $u: D_{*} \rightarrow \mathbb{R}$ be a harmonic function such that

$$
\lim _{w \rightarrow \zeta} u(w)=\varphi\left(F_{*}^{-1}(\zeta)\right) \quad \forall \zeta \in \partial D^{*}
$$

whose existence is well-known, see, e.g., Section 3 of Chapter VI in [11].

Let $z_{0} \in D, B_{0}:=B\left(z_{0}, \varepsilon_{0}\right) \subseteq D$ for some $\varepsilon_{0}>0$. Then the domain $D_{0}=$ $F\left(B_{0}\right)$ is simply connected and hence there is a harmonic function $v(w)$ such that $h(w)=u(w)+i v(w)$ is a holomorphic function which is unique up to an additive constant, see, e.g., Theorem 1 in Section 7 of Chapter III, Part 3 in [15]. Note that $f_{0}:=\left.h \circ F\right|_{B_{0}}$ is a local regular solution of the Beltrami equation (1.1). Note that the function $h$ can be extended to a multi-valued analytic function $H$ in the domain $D_{*}$ and, thus, $H \circ F$ gives the desired multi-valued solutions of the Dirichlet problem (1.5) for the Beltrami equation (1.1).

In particular, by Lemmas 2.1 and 6.1 we obtain the following.

Theorem 8.1. Let $D$ be a bounded domain in $\mathbb{C}$ whose boundary consists of $n \geqslant 2$ mutually disjoint Jordan curves and $\mu: D \rightarrow \mathbb{C}$ be a measurable function 
with $|\mu(z)|<1$ a.e. and such that $K_{\mu}(z) \leqslant Q(z)$ a.e. in $D$ for a function $Q: \mathbb{C} \rightarrow[0, \infty]$ in $\operatorname{FMO}(\bar{D})$. Then the Beltrami equation (1.1) has a multi-valued solutions of the Dirichlet problem (1.5) for each continuous function $\varphi: \partial D \rightarrow \mathbb{R}$.

Corollary 8.1. In particular, the conclusion of Theorem 8.1 holds if every point $z_{0} \in \bar{D}$ is Lebesgue point of a locally integrable function $Q: \mathbb{C} \rightarrow[0, \infty]$ such that $K_{\mu}(z) \leqslant Q(z)$ a.e. in $D$.

We assume that $K_{\mu}$ is extended by zero outside of $D$ in the following theorems.

Corollary 8.2. Let $D$ be a bounded domain in $\mathbb{C}$ whose boundary consists of $n \geqslant 2$ mutually disjoint Jordan curves and $\mu: D \rightarrow \mathbb{C}$ be a measurable function such that

$$
\varlimsup_{\varepsilon \rightarrow 0} f_{B\left(z_{0}, \varepsilon\right)} K_{\mu}(z) d m(z)<\infty \quad \forall z_{0} \in \bar{D} .
$$

Then the Beltrami equation (1.1) has a multi-valued solutions of the Dirichlet problem (1.5) for each continuous function $\varphi: \partial D \rightarrow \mathbb{R}$.

Theorem 8.2. Let $D$ be a bounded domain in $\mathbb{C}$ whose boundary consists of $n \geqslant 2$ mutually disjoint Jordan curves and $\mu: D \rightarrow \mathbb{C}$ be a measurable function with $|\mu(z)|<1$ a.e. If $K_{\mu} \in L_{\mathrm{loc}}^{1}(D)$ and satisfies the condition

$$
\int_{0}^{\delta\left(z_{0}\right)} \frac{d r}{\left\|K_{\mu}\right\|_{1}\left(z_{0}, r\right)}=\infty
$$

for some $\delta\left(z_{0}\right) \in\left(0, d\left(z_{0}\right)\right)$ where $d\left(z_{0}\right)=\sup _{z \in D}\left|z-z_{0}\right|$ and

$$
\left\|K_{\mu}\right\|_{1}\left(z_{0}, r\right)=\int_{D \cap S\left(z_{0}, r\right)} K_{\mu}(z)|d z|,
$$

at each point $z_{0} \in \bar{D}$. Then the Beltrami equation (1.1) has a multi-valued solutions of the Dirichlet problem (1.5) for each continuous function $\varphi: \partial D \rightarrow \mathbb{R}$.

Corollary 8.3. Let $D$ be a bounded domain in $\mathbb{C}$ whose boundary consists of $n \geqslant 2$ mutually disjoint Jordan curves and $\mu: D \rightarrow \mathbb{C}$ be a measurable function such that

$$
k_{z_{0}}(\varepsilon)=O\left(\log \frac{1}{\varepsilon}\right) \quad \text { as } \quad \varepsilon \rightarrow 0 \quad \forall z_{0} \in \bar{D}
$$

where $k_{z_{0}}(\varepsilon)$ is the average of the function $K_{\mu}(z)$ over $S\left(z_{0}, \varepsilon\right)$. Then the Beltrami equation (1.1) has a multi-valued solutions of the Dirichlet problem (1.5) for each continuous function $\varphi: \partial D \rightarrow \mathbb{R}$.

Remark 8.1. In particular, the conclusion holds if

$$
K_{\mu}(z)=O\left(\log \frac{1}{\left|z-z_{0}\right|}\right) \quad \text { as } \quad z \rightarrow z_{0} \quad \forall z_{0} \in \bar{D}
$$


Theorem 8.3. Let $D$ be a bounded domain in $\mathbb{C}$ whose boundary consists of $n \geqslant 2$ mutually disjoint Jordan curves and $\mu: D \rightarrow \mathbb{C}$ be a measurable function such that $|\mu(z)|<1$ a.e. and

$$
\int_{D} \Phi\left(K_{\mu}(z)\right) d m(z)<\infty
$$

for a convex non-decreasing function $\Phi:[0, \infty] \rightarrow[0, \infty]$. If

$$
\int_{\delta}^{\infty} \frac{d \tau}{\tau \Phi^{-1}(\tau)}=\infty
$$

for some $\delta>\Phi(0)$. Then the Beltrami equation (1.1) has a multi-valued solutions of the Dirichlet problem (1.5) for each continuous function $\varphi: \partial D \rightarrow \mathbb{R}$.

Corollary 8.4. In particular, the conclusion of Theorem 8.3 holds if, for some $\alpha>0$,

$$
\int_{D} e^{\alpha K_{\mu}(z)} d m(z)<\infty .
$$

\section{References}

[1] Bojarski B.: Generalized solutions of a system of differential equations of the first order of the elliptic type with discontinuous coefficients. Mat. Sb. 43(85)(4), 451-503 (1957).

[2] Bojarski B., Gutlyanskii V. and Ryazanov V.: General Beltrami equations and BMO. Ukr. Mat. Visn. 5(3), 305-326 (2008); transl. in Ukrain. Math. Bull. 5(3), 305-326 (2008).

[3] Bojarski B., Gutlyanskii V. and Ryazanov V.: On the Beltrami equations with two characteristics. Complex Var. Elliptic Equ. 54(10), 935-950 (2009).

[4] Bojarski B., Gutlyanskii V. and Ryazanov V.: On Integral Conditions for the General Beltrami Equations. Complex Anal Oper. Theory. 5(3), 835-845 (2011).

[5] Brezis H., Nirenberg L.: Degree theory and BMO. I. Compact manifolds without boundaries. Selecta Math. (N.S.) 1(2), 197-263 (1995).

[6] Dybov Yu.: On regular solutions of the Dirichlet problem for the Beltrami equations. Complex Var. Elliptic Equ. 55(12), 1099-1116 (2010). 
[7] Federer H.: Geometric Measure Theory. Springer-Verlag, Berlin (1969).

[8] Fuglede B.: Extremal length and functional completion. Acta Math. 98, 171-219 (1957).

[9] Gehring F.W., Lehto O.: On the total differentiability of functions of a complex variable. Ann. Acad. Sci. Fenn. Ser. A1. Math. 272, 1-9 (1959).

[10] Gehring F.W., Martio O.: Quasiextremal distance domains and extension of quasiconformal mappings. J. Anal. Math. 45, 181-206 (1985).

[11] Goluzin G. M.: Geometric Theory of Functions of a Complex Variable (in Russian), 2nd ed., Izdat. Akad. Nauk SSSR, 1966; Engl. transl. in Amer. Math. Soc. Transl., v. 26, 1969, pp. 136140, 165170, 375379, 599, 643644;

[12] Gutlyanskii V., Ryazanov V., Srebro U. and Yakubov E.: On recent advances in the degenerate Beltrami equations. Ukr. Mat. Visn. 7(4), 467-515 (2010); transl. in J. Math. Sci., 175(4), 413-449 (2011).

[13] Heinonen J., Kilpelainen T., Martio O.: Nonlinear Potential Theory of Degenerate Elliptic Equations. Clarendon Press, Oxford Univ. Press (1993).

[14] Hesse J.: A p-extremal length and p-capacity equality. Ark. Mat. 13, 131-144 (1975).

[15] Hurwitz A., Courant R.: The Function theory. Nauka, Moscow (1968) [in Russian].

[16] John F., Nirenberg L.: On functions of bounded mean oscillation. Comm. Pure Appl. Math. 14, 415-426 (1961).

[17] Ignat'ev A., Ryazanov V.: Finite mean oscillation in the mapping theory. Ukr. Mat. Vis. 2(3), 395-417, (2005) [in Russian]; transl. in Ukrain. Math. Bull. 2(3), 403-424, (2005).

[18] Ignat'ev A. and Ryazanov V.: Finite mean oscillation in the mapping theory. Reports of the Dept. of Math., University of Helsinki, Preprint 332, October 2002, 1-17.

[19] Kovtonyuk D., Ryazanov V.: On boundaries of space domains. Proc. Inst. Appl. Math. \& Mech. NAS of Ukraine. 13, 110-120 (2006) [in Russian].

[20] Kovtonyuk D., Ryazanov V.: On the theory of lower Q-homeomorphisms. Ukr. Mat. Visn. 5(2), 159-184 (2008); transl. in Ukrain. Math. Bull. 5(2), 157-181 (2008).

[21] Krushkal' S.L., Kühnau R.: Quasiconformal mappings: new methods and applications, Novosibirsk, Nauka (1984) (in Russian). 
[22] Martio O., Ryazanov V., Srebro U., Yakubov E.: Q-homeomorphisms. Contemporary Math. 364, 193-203 (2004).

[23] Martio O., Ryazanov V., Srebro U., Yakubov E.: On Q-homeomorphisms. Ann. Acad. Sci. Fenn. Ser. A1 Math. 30, 49-69 (2005).

[24] Martio O., Ryazanov V., Srebro U., Yakubov E.: Moduli in Modern Mapping Theory. Springer Monographs in Mathematics. Springer, New York (2009).

[25] Martio O., Sarvas J.: Injectivity theorems in plane and space. Ann. Acad. Sci. Fenn. Ser. A1 Math. 4, 384-401 (1978/1979).

[26] O. Martio, M. Vuorinen: Whitney cubes, $p$-capacity and Minkowski content. Expo. Math. 5, 17-40 (1987).

[27] Maz'ya V.: Sobolev Spaces. Springer-Verlag, Berlin (1985).

[28] Menchoff D.: Sur les differentielles totales des fonctions univalentes. Math. Ann. 105, 75-85 (1931).

[29] Näkki R.: Boundary behavior of quasiconformal mappings in $n$-space. Ann. Acad. Sci. Fenn. Ser. A1 Math. 484, 1-50 (1970).

[30] Ransford Th.: Potential Theory in the Complex Plane, Cambridge, Cambridge Univ. Press (1995).

[31] Ryazanov V., Salimov R.: Weakly flat spaces and boundaries in the mapping theory // Ukr. Mat. Visn. 4(2), 199-234 (2007); transl. in Ukrain. Math. Bull. 4(2), 199-233 (2007).

[32] Ryazanov V., Srebro U. and Yakubov E.: BMO-quasiconformal mappings, J. Anal. Math. 83, 1-20 (2001).

[33] Ryazanov V., Srebro U., Yakubov E.: Plane mappings with dilatation dominated by functions of bounded mean oscillation. Sib. Adv. Math. 11(2), 94-130 (2001).

[34] Ryazanov V., Srebro U., Yakubov E.: Beltrami equation and FMO functions. Contemp. Math. 382, 357-364 (2005).

[35] Ryazanov V., Srebro U. and Yakubov E.: Finite mean oscillation and the Beltrami equation. Israel J. Math. 153, 247-266 (2006).

[36] Ryazanov V., U. Srebro and Yakubov E.: On strong solutions of the Beltrami equations. Complex Var. Elliptic Equ. 55(1-3), 219-236 (2010).

[37] Ryazanov V., Srebro U., Yakubov E.: Integral conditions in the theory of the Beltrami equations, arXiv: 1001.2821v11 [math.CV] 12 Apr. 2010, 1-26. 
[38] Ryazanov V., Srebro U., Yakubov E.: Integral conditions in the theory of the Beltrami equations, Complex Var. Elliptic Equ. - 2011. - DOI: 10.1080/17476933.2010.534790.

[39] Ryazanov V., Srebro U., Yakubov E.: Integral conditions in the mapping theory. Ukr. Mat. Visn. 7, 73-87 (2010); transl. in Math. Sci. J.

[40] Reimann H.M., Rychener T.: Funktionen Beschränkter Mittlerer Oscillation. Lecture Notes in Math. 487 (1975).

[41] Saks S.: Theory of the Integral, Dover Publ. Inc., New York, 1964.

[42] Srebro U. and Yakubov E.: The Beltrami equation. Handbook in Complex Analysis: Geometric function theory. 2, 555-597, Elseiver, Amsterdam (2005).

[43] Stoilow S.: Lecons sur les Principes Topologue de le Theorie des Fonctions Analytique. Gauthier-Villars (1938). Riemann, Gauthier-Villars, Paris (1956) [in French].

[44] Väisälä J.: Lectures on $n$-Dimensional Quasiconformal Mappings. Lecture Notes in Math. 229. Springer-Verlag, Berlin etc. (1971).

[45] I.N. Vekua. Generalized analytic functions. Pergamon Press, London, 1962.

[46] Wilder R.L.: Topology of Manifolds. AMS, New York (1949).

[47] Ziemer W.P.: Extremal length and conformal capacity. Trans. Amer. Math. Soc. 126(3), 460-473 (1967).

Denis Kovtonyuk, Igor Petkov, Vladimir Ryazanov, Ruslan Salimov, Institute of Applied Mathematics and Mechanics, National Academy of Sciences of Ukraine, 74 Roze Luxemburg str., 83114 Donetsk, UKRAINE denis_kovtonyuk@bk.ru, igorpetkov@i.ua, vlryazanov1@rambler.ru,vl.ryazanov1@gmail.com, salimov07@rambler.ru 